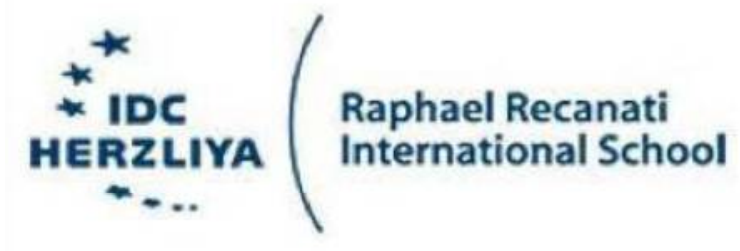

Seminar Paper

Advanced Topics in Social Network Analysis

\title{
Homophily and Purchase \\ Intention
}

Submitted by:

Sharon Barzily and Valerie Ackerman

July 16th, 2015 


\section{Introduction}

With the emergence and pervasiveness of online social networks, marketers and corporations constantly look for different ways to exploit their potential in terms of promoting products and brands. There has been increasing interest in studying what influences purchase intentions on online social networks in hopes to more accurately target consumers.

This seminar paper will study the correlation between different types of homophily and attitudes towards purchase intentions. Homophily means how similar two or more people are and refers to the idea that people are more likely to associate themselves with people that are similar to them than vice versa. More specifically, this paper will attempt to determine which are the types of homophily (i.e. gender, income etc.) that better estimate what products, two or more people would commonly be interested in.

The Interdisciplinary Center Herzliya offers a course called MiLab Studio which is modeled after the Massachusetts Institute of Technology (MIT) Media Lab. As members of this course, we were asked to create and develop a mobile application that enhanced communication between strangers in the same location. Our mobile application, Divvy, is an app that allows shoppers to find someone that would like to share the same 'buy one, get one' deals they are interested in, and ultimately save money. The motivation behind this research is to gain more insight about what specific similarities (homophily) between two customers will determine if those two people are likely to share the same deal. If the application will be able to recognize similarities between users, it could push certain deals towards those users and facilitate communication that would have otherwise been non-existent between those two shoppers.

In general this study could potentially help companies' marketing departments gain perspective into which elements they should take into consideration when planning an online marketing strategy. More specifically, companies could utilize certain shared attributes between two or more people, and construct a successful strategy to sell a product. This way, companies can devise a target audience that is 
relevant to the product rather than targeting the person's entire network as is commonly practiced today.

The goal of this study is therefore to define which are the types of homophily that will better correlate shoppers attitudes towards purchase intentions, thus allowing the Divvy application to exercise its full potential and successfully promote products of mutual interest to its users.

\section{Literature review}

Although the phenomenon of modern social network analysis is a relatively new field in the academic world, especially since the advent of the Internet, analyzing patterns in social networks has been around for many years. This can be seen in old descent lists included in the bible, as well as in ancient education systems that required members of different cultures to memorize many generations before them (Freeman, 1996).

In today's world of social science, social network analysis is described as the study of interaction amongst social individuals in which researchers study the patterned links between two or more actors, groups, and organizations and its consequences (Freeman, 1996; Hayat \& Mo, 2015). The paradigm of social network analysis serves as the basic framework for this seminar paper. The paradigm is motivated by structured intuition, grounded in empirical data, and relies on graphic imagery and computational models (Freeman, 1996; Hayat \& Lyons, 2010). A key precursor to social network analysis was German sociologist, Georg Simmel, who believed "societies exist where a number of individuals enter into interaction" (Simmel $1908 / 1971$ p.23). Simmel's studies have contributed to the general recognition of the foundation for social network analysis.

SNA has been predominant in the study of online social networks, also known as social network sites (SNS). These sites have been drawing in attention from researchers and marketers who are interested in the site's affordances and reach capability (Boyd \& Ellison, 2007; Hayat, Samuel-Azran \& Galily, 2016; Lesser, Hayat, \& Elovici, 2016). Boyd and Ellison (2007) coined and defined SNS as online services that allow people to create a public profile in a restricted environment, and to form and review a list of shared connections. These connections may vary from network to 
network. Marketers are putting increasing efforts into tracking consumer behavior through SNS, making SNS a valuable tool that can be used to create a social network based strategy that controls the service experience for their customers.

Due to increasing interest about online networks, many related questions have been raised about consumer behavior and purchasing intentions on social networking sites. This is valuable because purchase intentions can usually lead to actual purchase. According to Azjen (2002), behavioral intention is guided by three factors; belief about the outcome of the behavior, belief of the normative expectation of others' actions and belief about external factors which may help or hinder the completion of an action. Based on this definition, (Zainuddin \& Qader, 2011) defined purchase intentions as "a person's plan to engage in some action within a specified period of time and the probability that he or she will perform a behavior." There has been past research regarding various determinants of purchase intentions. For example, in a 2001 study it was found that a person's attitude towards a product almost exclusively reflected their purchase intentions (Bredahl, 2001). The overall attitude of the product which determined the purchase intentions were ultimately affected by the trustworthiness of the product itself. Another study found that the perceived value of a product induces the purchase intentions of a customer. The value of a product consisted of price and quality measurements, and the higher the value of a product, the higher the purchase intention of a customer (Chang \& Wildt, 1994).

Another key factor that has been found to influence purchase intentions within social networks is homophily. Homophily is the fundamental idea that people are more likely to associate or group themselves with people who are more similar to them, than with people they have no similarities with (McPherson, Smith-Lovin, \& Cook, 2001). A homophilous network will limit your social world and restrict the information you receive since most of the members in your network share your characteristics. When you are distant with another person in terms of homophily, you will be more distant in terms of your network as well (McPherson, Smith-Lovin, \& Cook, 2001). For example Beth Wellman (1929) found that kids at school are more likely to create friendships with other kids that have similar demographic 
characteristics such as height, school grades, IQ, physical education and more, faster than they would with kids that are dissimilar to them (Freeman, 1996).

There has been an abundant amount of research involving the differentiation between homophily and influence, and it has since been regarded as an extremely difficult task to measure. There is little sure way of knowing whether people are friends with who they are because they have similarities with each other, or because they influence their friends, creating more similarities between them over time. Aral, Muchnik, and Sundararajan (2009) studied the difference between social influence from homophily in networks, and found that when comparing the two, homophily accounted for a big part of what one normally would thing was social influence. Although social influence is important to mention when discussing homophily, this paper will not address the matter of social influence, rather focus on different types of homophily.

Various studies have addressed the different types of homophily such as intrapersonal characteristics, behavioral, and sociodemographic (McPherson, SmithLovin, \& Cook, 2001) and measured the relationship with different variables. A study by Thelwall (2008) looked at participant's active friends on MySpace and studied homophily in their network regarding race, gender, marital status, sexual orientation ethnicity, reasons for joining MySpace, attitudes towards children, age and religion. He found that the only element of homophily which was not significant was gender, all other factors were found to be homophilous within the network. In the same way, it has been found that homophily in race and ethnicity creates the biggest gap in our personal surroundings, with age, religion, education, occupation, and gender following respectively (McPherson, Smith-Lovin, and Cook, 2001). Homophily has also been proven to play a significant role in the world of online dating. A study found that members of online dating sites decide their preferences based on homophily in areas such as stage of life, marital status, want for children, physical attractiveness, and smoking habits (Fiore \& Donath, 2005).

Furthermore, homophily is a vital concept that gives an explanation as to why trust relations are formed (Mo, Hayat, \& Wellman, 2015). Trust can serve great important for those who are seeking information they can depend on from the online 
web (Tang, Gao, Hu, \& Liu, 2013). As mentioned before, trust also ties in to purchase intentions, as the amount of trust towards a product will change a person's attitude about their intentions of buying that product (Chang \& Wildt, 1994).

Taking into account the results from past research on homophily, it can be concluded that homophily plays a crucial role in defining and influencing a network structure. Homophily accounts for a great part of a person's network and as mentioned before different types of homophily have been studied and found significant in relationship forming. (Thelwall, 2008). In regards to purchase intentions, people that are close in network distance frequently make similar purchases, and these similarities can be explained through homophily. Evidence has shown that homophily affects purchase intentions and product choices (Ma, Krishnan \& Montgomery, 2015). This paper will analyze in a quantitative manner the different types of homophily within a person's Facebook network and its correlation with attitudes towards purchase intentions, in hopes to find specific types of homophily that will be used to estimate which of the same products will two or more people be interested in. The results of this research could be useful regarding Divvy's future (mobile application), in the sense that it could allow us to push certain products towards two people based on their homophily, in hopes that they will both be interested in the same deal. It is hypothesized that (1) ethnicity homophily will have a strong correlation with attitudes towards purchase intentions of facebook users(2) gender homophily will have a strong correlation with attitudes towards purchase intentions of facebook users and (3) income homophily will have a strong correlation with attitudes towards purchase intentions of facebook users. (4) age homophily will have a strong correlation with attitudes towards purchase intentions of facebook users. (5) hometown homophily will have a strong correlation with attitudes towards purchase intentions of facebook users. (6) Current city homophily will have a strong correlation with attitudes towards purchase intentions of facebook users. (7) Education level homophily will have a strong correlation with attitudes towards purchase intentions of facebook users. These variables were selected according to the existing literature as being correlated with other behavior changes and also taking into account the information about variables that Facebook provides. It was important 
to test for those variables (hometown, current city and education level) due to the fact that for the apps future, Facebook could be linked to it as a form of "login", and data drawn from it may be relevant for further advertisements of products.

\section{Method}

\section{Sample}

33 respondents were gathered, 18 being women and 15 men. All respondents were students from IDC Herzliya International School, from different tracks of studies. The sample was not gathered randomly rather, by convenience. Moreover the sample for this research reflects Divvy's approximate target audience, focusing on college students both male and female. It's been shown that homophily works for different groups of society, thus there is no reason why a sample of this nature wouldn't show homophily. It can be assumed that respondents come from different countries due to the fact that IDC is an International school. The name of each respondent's hometown was not collected because we were more interested in identifying homophily of hometowns, rather than specific names of cities or countries.

\section{Tools}

A survey was created specifically for this study including 9 items in order to measure homophily and attitudes towards purchase intentions quantitatively. Attitudes towards purchase intentions was operationalized by asking participants if they are likely to be influenced in their purchase intention after being recommended to buy a clothing item by each of the 3 names they listed. Although we would have liked to follow up on actual purchase intentions, we were unable to observe actual purchasing and instead used information from the survey to substantiate our dependent variable. As we have previously discussed the variables; ethnicity, education level, age, hometown, income, gender, closeness, and current city are found to be relevant in homophily studies which serves as an explanation for why we chose to include these variables in our survey. Participants were asked to name up to 3 individual ties from their network, who they would listen to if they would recommend them a clothing item. 
Each dyad (i.e. participant and tie 1) was observed for homophily among the listed variables and measured accordingly. This was done separately for each of the three ties that the participant named. A 5 point likert scale was used to measure how likely they believed they were to purchase something from each of the people they had previously named, if they were to recommend them a clothing item. Moreover, a 5 point likert scale was also used for participants to rate how close (closeness variable) to each of these 3 people they feel they are, where 1 means very close and 5 means not close at all. Later they were asked to answer if they had the same, or not the same, ethnicity, education level, age, hometown, income, gender, and current city as each of the 3 people they had listed to observe dyadic homophily between each participant and their ties. See appendix A for complete survey.

\section{Procedure}

The web-based survey generator 'SurveyMonkey' was used to collect the data for this paper. The link of the survey was sent in a private message to the participants via Facebook by the researchers. Participants responded at their own pace and were not given a deadline to complete the survey. After messaging all 33 respondents and acquiring a $68 \%$ response rate, the survey link was officially closed after a period of three days. Participants were asked to notify the researchers upon completion. This served as a recordable way to keep track of gender balance within the sample.

\section{Results}

There were a total of 33 respondents from IDC Herzliya International School participated in the survey. It was found that the mean distribution for the independent variable of closeness is 3.97 . Out of the 33 respondents, $57 \%$ answered that they were very close to the person they would listen to if they were recommended a clothing item by them. Additionally, the mean distribution for the dependent variable of purchase intentions is 4.31 . This includes $47.9 \%$ of respondents who were very likely to purchase a clothing item from the person they listed. See figure 1.0, 1.1, \& 1.2. 

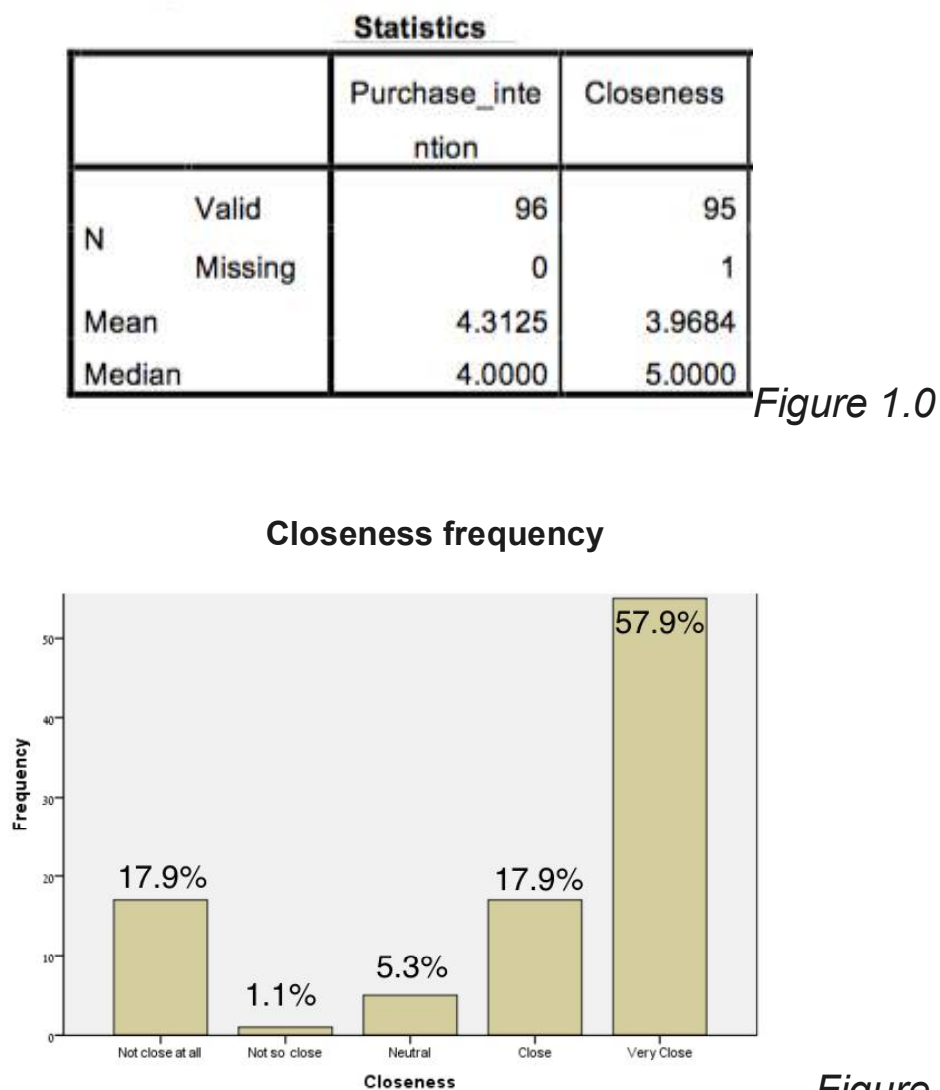

Figure 1.1

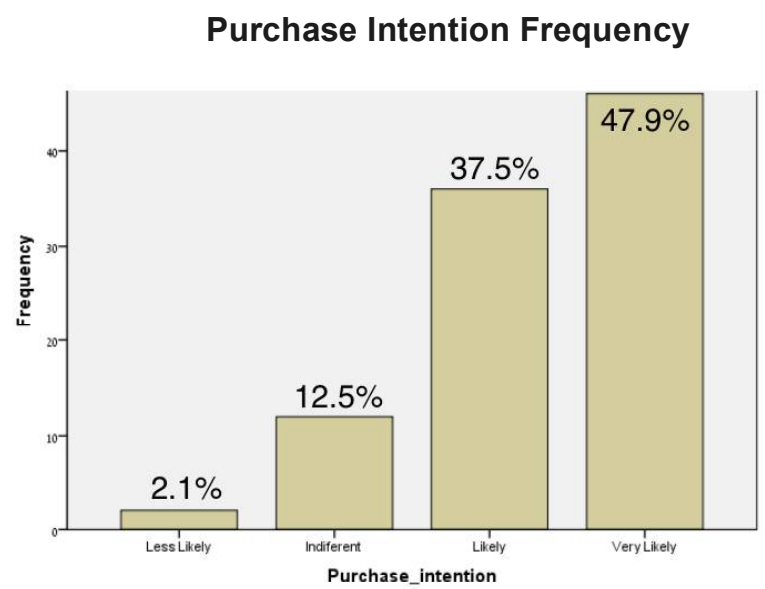

Figure 1.2 
In order to analyze the data, Spearman correlation test and Multiple Linear Regression was used. All six hypotheses were not supported, and only H6 showed significant correlation.

In order to examine hypotheses H6 Spearman's Rank Order Correlation was conducted, with current city homophily as the independent variable and purchase intention as the dependent variable. Consistent with the hypothesis we found significant negative and weak relationship between the variables (Rs= $-.209, P<$ 0.05). This means that when current city homophily increases purchase intention decreases and vice versa. Therefore hypothesis 6 is rejected.

Other significant correlations that were not hypothesized on were found between independent variables:

Spearman's Rank Order Correlation was conducted, with current city homophily and education level homophily variables. We found significant positive weak correlation between the variables $(R s=.335, P<0.05)$. This means that when current city homophily increases education level homophily increases and vice versa.

Additionally Spearman's Rank Order Correlation was conducted, with ethnicity homophily and education level homophily variables. We found significant negative and weak relationship between the variables (Rs $=-.210, P<0.05)$. This means that when current city homophily increases ethnicity homophily decreases and vice versa.

Moreover to predict product intentions on the basis of current city homophily, ethnicity homophily, education level homophily, age homophily, income homophily and hometown homophily, Multiple linear regression analysis was conducted. The regression model was not found to be significant.

Although the regression model was not found statistically significant, on a descriptive level we are able to assess the three independent variables that are most strongly correlated with the dependent variable. Due to the fact the independent variables were measured using different scales, the Beta coefficients were used as a way to normalize the data and enabled us to identify the strongest correlations. Current city homophily had the strongest correlation on purchase intentions of -.292. Education level homophily was second strongest with a correlation of .186. Closeness was the third strongest correlation with .059. 


\section{Discussion}

Even though the hypotheses were not supported at a statistical level, at a descriptive level, it was found that there are some types of homophily that may better explain purchase intentions than others. Survey results indicated that current city homophily has a negative correlation with purchase intentions, meaning the more homophilous your current city is to that of another person, the less likely your purchase intentions will be influenced by this person. Although this did not support our hypothesis, a possible explanation for this finding could have something to do with the fact that all of the respondents attend an international university, and it is likely that the close ties in their network (i.e. family) are situated abroad.

There were no significant results found for the remaining hypotheses, which could be due in part to the small sample size. This limitation should serve as a guide for future research, indicating that a larger sample size could assist in finding more significant results. Income level homophily did not have any significant correlation to purchase intentions, thus $\mathrm{H} 3$ was not supported. This may possibly be attributed to the fact that close ties in one's network can include family members such as parents who are likely to have different income levels than university students. Therefore, in the future it would be beneficial to look into other socioeconomic variables aside from income level, such as monthly expenses, to better predict if socioeconomic variables can influence purchase intentions.

Although it was found that age was relevant to a person's network on MySpace and therefore assumed to be relevant to a person's general network, (Thelwall 2008), the age variable may have not been relevant in our sample because respondent's close ties may have included family members that are possibly much older than them. Moreover, due to the fact that in Israel some people need to serve in the military, and others come straight after high school from abroad, this may variate the ages of people within the same classroom. Although no significant results were found regarding age and purchase intentions, future research should expand the sample size in order to observe if significant effects of age on purchase intentions 
exist. This information could be utilized by mobile applications like Divvy, to help target the relevant audience and suggest products that will be mutually desired.

Hometown homophily and ethnicity were not significant for the evident reason that our respondents attended an international school and came from many different parts of the world.

One potential explanation that gender homophily was not found significant, may be because men are more likely to ask women for opinions and women more likely to ask other women for fashion advice in a general sense. In the future it might be valuable to test each gender separately to gain better insight on gender homophily and purchase intentions. Also, expanding upon the items for purchase intentions could provide more accurate results. We chose a clothing item due to time constraints and also because we believed a clothing item is something that can be related to the majority of the population and by both genders. Having said that, there may be more gender neutral categories to select items from that are worth being explored.

In terms of the multiple linear regression, although no significant results were found, on a descriptive level, we were able to identify three variables that were the most strongly correlated with purchase intentions. Results showed that $29.2 \%$ of the intention to purchase can be explained by the current city homophily, $18.6 \%$ of the intention to purchase can be explained by the education level homophily, and $5.9 \%$ of the intention to purchase can be explained by the closeness variable.

The practical implications of identifying the three variables, current city homophily, education level homophily, and closeness can include serving as a valuable insight for our mobile application, Divvy. When deciding what deals to send the target users, it might be useful to identify these types of homophily among the users, in hopes of increasing the amount of successful matches between users on the application. In addition, advertisers in general may be able to look into these types of homophily to better their marketing efforts and influence purchase intentions by targeting the right audience.

In conclusion, in this study 33 university students from the Interdisciplinary Center Herzliya, were surveyed to examine if certain types of homophily are correlated with purchase intentions in order to potentially use this insight towards the 
improvement of the mobile application, Divvy. Divvy is an app that allows shoppers to find someone that would like to share the same 'buy one, get one' deals they are interested in, and ultimately save money. It was hypothesized that all variables (ethnicity homophily, gender homophily, income level homophily, age homophily. hometown homophily, current city homophily, and education level homophily) would have a strong positive correlation with purchase intentions. Although the results did not show statistical significance, on a descriptive level we were able to identify the three variables with the strongest correlation to purchase intentions. In general, this study could potentially assist companies' advertising efforts by targeting the right audience and devising a successful marketing campaign based on consumers with mutual characteristics. Furthermore, this study could serve future research in exploring deeper what types of homophily may affect purchase intentions. 


\section{References}

Aral, S., Muchnik, L., \& Sundararajan, A., (2009). Distinguishing influence-based contagion from homophily-driven diffusion in dynamic networks. PNAS.

Azjen, I. (2002). Perceived behavioral control, self-efficacy, locus of control, and the theory of planned behavior. Journal of Applied Social Psychology 32(4), 665-583

Boyd, D., \& Ellison, N. (2007). Social network sites: Definition, history, and scholarship Journal of Computer-Mediated Communication 13, (1), 210-230.

Bredahl, L. (2001). Determinants of consumer attitudes and purchase intentions with regard to genetically modified food-results of a cross-national survey. Journal of consumer policy, 24(1), 23-61.

Chang T., \& Wildt, A. (1994). Price product information and purchase intention: An empirical Study. Journal of the Academy of Marketing Science. 22(1), 16-27.

Fiore, A., \& Donath, J. (2005). Homophily in online dating: when do you like someone like yourself?. In Extended Abstracts on Human Factors in Computing Systems, 1371-1374.

Freeman, L. (1996). Some Antecedents of Social Network Analysis1 19(1), 39-42.

Hayat, Z., \& Lyons, K. (2010, November). The evolution of the CASCON community: a social network analysis. In Proceedings of the 2010 Conference of the Center for Advanced Studies on Collaborative Research (pp. 1-12). IBM Corp.

Hayat, T., \& Mo, G. Y. (2015). Advice giving and receiving within a research network. American Behavioral Scientist, 59(5), 582-598.

Hayat, T., Samuel-Azran, T.,\& Galily, Y. (2016). Al-Jazeera Sport's US Twitter followers: sport-politics nexus?. Online Information Review, 40(6), 785-797.

Tang, J., Gao, H., Hu, X., \& Liu, H. (2013). Exploiting Homophily Effect for Trust Prediction. Proceedings of the sixth ACM international conference on Web search and data mining, 53-62.

Thelwall, M., (2008). Homophily in MySpace. Journal of the American Society for Information Science and Technology. 60(2), 219-231.

Lesser, O., Hayat, T., \& Elovici, Y. (2017). The role of network setting and gender in online content popularity. Information, Communication \& Society, 20(11), 16071624.

Ma, L., Krishnan R., \& Montgomery, A. (2015). Latent homophily or social influence? An empirical analysis of purchase within a social network. Management Science 61(2), 454-473.

McPherson, M., Smith-Lovin, L., \& Cook, J. M. (2001). Birds of a feather: Homophily in social networks. Annual review of sociology, 415-444.

Mo, G. Y., Hayat, Z., \& Wellman, B. (2015). How far can scholarly networks go? Examining the relationships between distance, disciplines, motivations, and clusters. In Communication and Information Technologies Annual (pp. 107-133). Emerald Group Publishing Limited.

Simmel, G., (1908/1971). On Individuality and Social Forms. Chicago:University of Chicago.

Wellman B. (1929). The school child's choice of companions. The Journal of Educational Research 14,126-32

Zainuddin I., \& Qader, Y. (2011). The impact of media exposure on intention to purchase green electronic products amongst lecturers. International Journal of Business and Management 6(3) 